\title{
Anti-hypoglycemic and hepatocyte-protective effects of hyperoside from Zanthoxylum bungeanum leaves in mice with high-carbohydrate/high-fat diet and alloxan-induced diabetes
}

\author{
YALI ZHANG $^{1}$, MIMI WANG ${ }^{1}$, HUANHUAN DONG $^{1}$, XIAOMIN YU ${ }^{1}$ and JINGFANG ZHANG ${ }^{2}$ \\ ${ }^{1}$ Key Laboratory of Biomedical Information Engineering of Ministry of Education, \\ School of Life Science and Technology, Xi'an Jiaotong University, Xi'an, Shaanxi 710049; \\ ${ }^{2}$ College of Forestry, Northwest A\&F University, Xianyang, Shaanxi 712100, P.R. China
}

Received April 11, 2017; Accepted October 17, 2017

DOI: $10.3892 /$ ijmm.2017.3211

\begin{abstract}
The development of diabetes mellitus (DM) is accompanied by hyperglycemia-induced oxidative stress. Hyperoside is a major bioactive component in Zanthoxylum bungeanum leaves (HZL) and is a natural antioxidant. However, the effects of HZL on DM and its mechanisms of action remain undefined. The present study evaluated the anti-hypoglycemic and hepatocyte-protective effects of HZL in mice with diabetes induced by a high-carbohydrate/high-fat diet (HFD) and alloxan. We also aimed to eludicate the underlying mechanisms. Our resutls demonstrated that the administration of HZL significantly reduced body weight gain, serum glucose levels and insulin levels in diabetic mice compared with the vehicle-treated mice. In addition, the levels of dyslipidemia markers including total cholesterol, triglyceride and low-density lipoprotein cholesterol in the HFD-treated mice were markedly decreased. Further experiments using hepatocytes from mice revealed that HZL significantly attenuated
\end{abstract}

Correspondence to: Professor Jingfang Zhang, College of Forestry, Northwest A\&F University, 3 Taicheng Road, Yangling, Xianyang, Shaanxi 712100, P.R. China

E-mail: zjf19890117@163.com

Abbreviations: HZL, hyperoside from Zanthoxylum bungeanum leaves; DM, diabetes mellitus; HFD, high-carbohydrate/high-fat diet; $\mathrm{TC}$, total cholesterol; TG, triglyceride; LDL-C, low-density lipoprotein cholesterol; HDL-C, high-density lipoprotein cholesterol; ALT, alanine aminotransferase; AST, aspartate aminotransferase; $\mathrm{NO}$, nitric oxide; iNOS, inducible nitric oxide synthase; SOD, superoxide dismutase; GPx, glutathione peroxidase; MDA, malondialdehyde; RT-qPCR, reverse transcription-quantitative polymerase chain reaction; OGTT, oral glucose tolerance test; ROS, reactive oxygen species

Key words: hyperoside, Zanthoxylum bungeanum leaves, nuclear factor- $\kappa \mathrm{B}$, mitogen-associated protein kinase, diabetes mellitus, liver injury liver injury associated with DM compared with vehicle treatment, as evidenced by lower levels of alanine aminotransferase and aspartate aminotransferase in serum and by lower levels of lipid peroxidation, nitric oxide content and inducible nitric oxide synthase activity in liver tissues. Nuclear factor- $\kappa \mathrm{B}$ (NF- $\mathrm{KB}$ ) and mitogen-associated protein kinase (MAPK) signaling pathways were investigated to elucidate the molecular mechanisms responsible for the protective effects of HZL against diabetic liver injury. The results indicated that HZL inhibited the phosphorylation of p65/NF- $\mathrm{BB}$, MAPK (including p38, JNK and ERK1/2) and activating transcription factor 3 protein expression, with an additional suppression of Bax, cytochrome $c$, caspase- 9 and caspase- 3 in the liver tissues of diabetic mice. Taken together, our findings suggest that HZL, which was effective in inhibiting oxidative stress-related pathways may be beneficial for use in the treatment of DM.

\section{Introduction}

Diabetes mellitus $(\mathrm{DM})$ is a metabolic disorder characterized by chronic hyperglycemia resulting from a defect in insulin metabolism and impaired function of carbohydrate, lipid and protein metabolism that lead to long-term complications. $\mathrm{DM}$ is associated with the generation of reactive oxygen species (ROS), which cause oxidative damage, particularly to the liver, kidney, eyes, small and large blood vessels, immune and gastrointestinal systems (1-3). The liver, an insulin-dependent organ, plays a pivotal role in glucose and lipid homeostasis, and is severely affected during the progression of DM $(4,5)$. Furthermore, the liver is the focal organ involved in oxidizing and detoxifying processes, as well as free radical reactions (6-8). Accumulating literature reviews suggest that enhanced oxidative stress and significant reduced antioxidant defenses are considered to play an important role in diabetic liver injury $(9,10)$.

Zanthoxylum bungeanum ( $Z$. bungeanum) belongs to the Zanthoxylum genus of the Rutaceae family. The fruit of $Z$. bungeanum, locally called 'Huajiao', is one of the best-known and widely used plants in traditional Chinese medicine. Young $Z$. bungeanum leaves have been used as foodstuffs, and mature $Z$. bungeanum leaves are considered 
carminative, stimulant and sudorific (11). The previous studies revealed that $Z$. bungeanum leaves are rich in flavonoids with good radical scavenging abilities $(12,13)$. Some studies have indicated that hyperoside is the major flavonoid of $Z$. bungeanum leaves (12-15). Pharmacological investigations have demonstrated that hyperoside have diverse biological activities such as antioxidant (12,16), anticancer (17), anti-inflammatory (18), anticoagulant (19) and cardioprotective activities (20). Moreover, hyperoside has been found to play crucial roles in rat lens aldose reductase inhibition (21). Previously, it has been reported that hyperoside has a pivotal role in blood glucose level in streptozotocin-induced hyperglycemia by improving the function of pancreatic islets, increasing glycolysis and decreasing gluconeogenesis (22). Hyperoside may be one of the primary glucosidase inhibitor constituents of Agrimonia pilosa Ledeb (23). However, only a few researchers have paid attention to investigating the preventive effects of hyperoside from $Z$. bungeanum leaves on diabetic liver injury. Currently, there are no available studies on the effects of hyperoside on diabetes induced by a highcarbohydrate/high-fat diet (HFD) and alloxan in mice, at least to the best of our knowledge. Since flavonol supplements in dietary food may evoke protective effects under oxidative stress, the present study determined the protective effects of hyperoside from Z. bungeanum leaves (HZL) on hyperglycemia and the liver damaged induced by HFD diet and alloxan in mice.

\section{Materials and methods}

Reagents. Leaves of Z. bungeanum were collected in Shaanxi, China in August 2015 and identified by experts in the College of Forestry, Northwest A\&F University (Xian, China). Alloxan was purchased from Sigma-Aldrich; Merck KGaA (Darmstadt, China). Blood glucose was measured using kits from Shanghai Rongsheng Biotechnology Co., Ltd. (Shanghai, China). The levels of total cholesterol (TC, cat. no. A111-1), triglyceride (TG, cat. no. A110-1), low-density lipoprotein cholesterol (LDL-C, cat. no. A113-1), high-density lipoprotein cholesterol (HDL-C, cat. no. A112-1), nitric oxide (NO, cat. no. A012-1), malondialdehyde (MDA, cat. no. A003-1), and the activities of $\mathrm{Na}^{+} / \mathrm{K}^{+}$ATPase (cat. no. A070-2), inducible nitric oxide synthase (iNOS, cat. no. A014-1-1), plasma alanine aminotransferase (ALT, cat. no. A009-2), aspartate aminotransferase (AST, cat.no. C010-2), superoxide dismutase (SOD, cat. no. A001-1-1), catalase (CAT, cat. no. A007-1-1), glutathione peroxidase (GPx, cat. no. A005) were detected using kits from the Nanjing Jiancheng Bioengineering Research Institute (Nanjing, China). Insulin levels were determined using a radioimmunoassay kit from Biosino Bio-Technology and Science, Inc. (Beijing, China). Rabbit anti-active p65, p-p65, p38, p-p38, JNK, p-JNK, ERK, p-ERK activating transcription factor 3 (ATF3), Bcl-2, Bax, cytochrome $c$ (Cyt c), caspase-9, and caspase-3 polyclonal antibodies were all purchased from Cell Signaling Technology, Inc. (Danvers, MA, USA); $\beta$-actin antibodies (cat. no. SC10731) were purchased from Santa Cruz Biotechnology, Inc. (Dallas, TX, USA). All the chemicals were of analytical grade. Hyperoside was isolated from $Z$. bungeanum leaves. Z. bungeanum leaves were soaked in a $70 \%$ ethanol solvent $(1: 10, \mathrm{w} / \mathrm{v})$ for $2.5 \mathrm{~h}$ and sonicated in an ultrasonic bath at $200 \mathrm{kHz}$ at $55^{\circ} \mathrm{C}$ for $45 \mathrm{~min}$. Samples were filtered, concentrated and dried using a rotary evaporator. The remaining ethanol crude extracts was further fractioned by column chromatography on silica gel (silica gel 200-300 mesh, 120x10 cm i.d., flow rate $10 \mathrm{ml} / \mathrm{min}$ ), successively eluting with petroleum ether, chloroform, ethyl acetate, acetone and methanol. A fraction of ethyl acetate was further separated and purified by high-performance liquid chromatography. The chromatographic conditions used were as follows: the column was a SB-C18 (250 mm x $4.6 \mathrm{~mm}$ i.d., $5 \mu \mathrm{m})$ at ambient temperature. The injection volume was $20 \mu \mathrm{l}$ and the detection wavelength was $254 \mathrm{~nm}$. The mobile phase consisted of water with $0.5 \%$ trifluoroacetic acid (solvent A) and acetonitrile with $0.5 \%$ trifluoroacetic acid (solvent $\mathrm{B}$ ). The flow rate was $0.8 \mathrm{ml} / \mathrm{min}$. The gradient program was set as follows: from 0 to $30 \mathrm{~min}$, eluent $\mathrm{B}$ was increased from 15 to $35 \%$; from 30 to $35 \mathrm{~min}$, eluent B was increased from 35 to $65 \%$; and from 35 to $55 \mathrm{~min}$, eluent B was increased from 65 to $100 \%$ and then maintained at $100 \%$ for $10-20 \mathrm{~min}$. Samples were filtered through a $0.22 \mu \mathrm{m}$ membrane filter prior to injection. The eluent was concentrated using the rotary vacuum evaporator and vacuum-dried to obtain hyperoside from HZL. The structure of the isolate was determined by reverse phase high-performance liquid chromatography in comparison with authentic hyperoside (National Institute for the Control of Pharmaceutical and Biological Products, Beijing, China).

Animals, diets and experimental design. Male Chinese Kunming mice (6-week-old, weighing 20 2 g, n=90) were obtained from the Experimental Animal Center of Xi'an Jiaotong University (Xi'an, China) and were allowed to acclimatize for 1 week before being randomly assigned to different experimental groups. All of the mice were maintained on a $12 \mathrm{~h}$ light/dark cycle on a standard chow diet until experimental analysis. The experimental animal protocol was approved by the Experimental Animal Ethics Committee of Xi'an Jiaotong University. The experimental procedures were carried out in accordance with international guidelines for the Care and Use of Experimental Animals.

After adaptation for 1 week, mice were randomly divided into 6 groups ( $\mathrm{n}=15$ in each group, 5 mice/cage) as follows: i) normal group; ii) normal $+200 \mathrm{mg} / \mathrm{kg}$ body weight (BW)/day HZL (HHZL) groups; iii) DM group: HFD-alloxan treatment (HFD, 52.6\% standard laboratory chow, $10 \%$ lard, $15 \%$ sucrose, $15 \%$ yolk powder, $5 \%$ casein, $1.2 \%$ cholesterol, $0.2 \%$ bile salt, $0.6 \%$ calcium bicarbonate); iv) $\mathrm{DM}+50 \mathrm{mg} / \mathrm{kg} \mathrm{BW} /$ day HZL (LHZL); v) DM + $100 \mathrm{mg} /$ $\mathrm{kg} \mathrm{BW} /$ day HZL (MHZL); and vi) DM + $200 \mathrm{mg} / \mathrm{kg} \mathrm{BW} /$ day HZL (HHZL). HZL was suspended in 1\% Tween-80 in water prior to administration. The mice in groups 2 and 4-6 received daily administrations by gastric gavage of different doses HZL, and mice in groups 1 and 3 were administered the vehicle ( $1 \%$ Tween- 80 in water, $10 \mathrm{ml} / \mathrm{kg} \mathrm{BW})$ once a day for 4 weeks. After 4 weeks of dietary manipulation, the groups of mice fed the HFD were injected intraperitoneally with $0.04 \%$ alloxan dissolved in sterile normal saline in a dose of $100 \mathrm{mg} / \mathrm{kg} \mathrm{BW}$ The mice were allowed to continue to feed on their respective diets till the experimental tenure. Water and food were available to the animals ad libitum. 
Body weight and food intake were recorded weekly. Two weeks after alloxan injection, the animals were anesthetized with isoflurane (3\%) after fasting for $8 \mathrm{~h}$, and blood samples were obtained by cardiac puncture. The mice were then sacrificed by cervical dislocation and liver were collected, weighed, frozen in liquid nitrogen and stored at $-80^{\circ} \mathrm{C}$ for further analysis.

Biochemical analyses of blood samples. Plasma was obtained by centrifuging the blood at $2,000 \mathrm{x}$ g for $15 \mathrm{~min}$ at $4^{\circ} \mathrm{C}$. Fasting blood glucose level was monitored periodically during the treatment with the tail prick method using kits based on the glucose oxidase method. The blood glucose level was expressed as mmol/l. Plasma insulin, TC, TG, HDL-C and LDL-C levels were measured by adhering tothe commercial kits' instructions.

The oral glucose tolerance test (OGTT) was performed at 14 days following alloxan treatment. Briefly, after fasting for $8 \mathrm{~h}$, blood was collected from the tail veins of all mice ( $0 \mathrm{~min})$. Immediately after blood collection, all mice received an intraperitoneal injection of glucose $(2 \mathrm{~g} / \mathrm{kg} \mathrm{BW})$. Blood samples were successively collected at the indicated time intervals $(0,30,60$ and $120 \mathrm{~min})$, and blood glucose levels were determined as mentioned above. The area under the curve (AUC) was calculated as the area under the glucose curve from 0 to 120 min multiplied by the minutes at the measured timepoints.

Liver oxidative stress levels test. Liver were thawed, weighed and homogenized with Tris- $\mathrm{HCl}(5 \mathrm{mM}$ containing $2 \mathrm{mM}$ EDTA, pH 7.4). Homogenates were centrifuged (1,500 x g, $10 \mathrm{~min}$, at $4^{\circ} \mathrm{C}$ ) and the supernatant was used immediately for the assays. $\mathrm{Na}^{+} / \mathrm{K}^{+}$ATPase activity, ALT activity, AST activities, lipid peroxidation product MDA level, SOD activity, CAT activity, GPx activity, iNOS activity and NO content were measured by following the commercial kits' instructions.

Western blot analysis of proteins. Whole protein lysates of liver tissue were extracted using radioimmunoprecipitation assay buffer [25 mM Tris-HCl (pH 7.6), $150 \mathrm{mM} \mathrm{NaCl}$, $1 \%$ NP-40, $1 \%$ sodium deoxycholate, $0.1 \%$ SDS; Thermo Fisher Scientific, Inc., Waltham, MA, USA] supplemented with $1 \%$ protease inhibitor cocktail and $1 \%$ phenylmethylsulfonyl fluoride. Protein concentrations were measured using a BCA Protein Assay kit (A045-3; Nanjing Jiancheng Bioengineering Research Institute). Aliquots containing $50 \mu \mathrm{g}$ of protein were loaded onto a into $8 \%$ SDS-PAGE gel, immunoblotted onto a polyvinylidene difluoride membrane (Takara Biotechnology Co., Ltd., Dalian, China), blocked for $1 \mathrm{~h}$ at room temperature with $5 \%$ bovine serum albumin in TBS buffer, and then incubated overnight $\left(4^{\circ} \mathrm{C}\right)$ with respective primary antibodies for anti-p65 (cat. no. 3034), anti-p-p65 (cat. no. 3033), anti-p38 (cat. no. 9212), anti-p-p38 (cat. no. 9216), anti-JNK (cat. no. 9252), anti-p-JNK (cat. no. 9255), anti-ERK1/2 (cat. no. 4695), anti-p-ERK1/2 (cat. no. 4370), anti-Bcl-2 (cat. no. 2876), anti-Bax (cat. no. 2772s), anti-Cyt c (cat. no. 4280s), anti-caspase-9 (cat. no. 95083) and anti-caspase-3 (cat. no. 9662s) (diluted 1:1,000; all from Cell Signaling Technology, Inc.) and anti-ATF3 (cat. no. ab7005, diluted 1:1,000; Abcam, Cambridge, UK). After being washed with TBS-T, membrane was incubated with secondary horseradish peroxidase-conjugated anti-rabbit IgGs (cat. no. ZB2301, diluted 1:2,000; Beijing Zhongshan Golden Bridge Biotechnology Co., Ltd., Beijing, China) for $2 \mathrm{~h}$ at room temperature. The bound complexes were detected by incubating with a chemiluminescence solution purchased from EMD Millipore (cat. no. WBKLS0050; Billerica, MA, USA) according to the manufacturer's instructions. Chemiluminescence was imaged on a Fujifilm LAS-3000 system (Fujifilm, Tokyo, Japan). The immunoblot bands were quantified by densitometry analysis, and the ratio to $\beta$-actin was calculated and presented, setting the values of normal diet fed mice as 1 .

Liver histological analysis. Liver tissues were fixed with $10 \%$ formalin and processed for paraffin embedding. Tissue sections were cut to $5 \mu \mathrm{m}$ and stained with hematoxylin and eosin for assessing histopathological changes. The degree of injury was assessed semi-quantitatively by the following criteria and scored based on the area affected: i) increased eosinophilic staining of the hepatocytes and the accumulation of erythrocytes in the sinusoids ( 0 , none; $1,<25 \% ; 2,25$ to $<50 \%$; 3,50 to $<75 \%$; and 4,75 to $100 \%$ ); ii) cellular vacuolization ( 0 , none; $1,<25 \%$; and $2,>25 \%$ ); and iii) cell lysis ( 0 , none; $1,<25 \%$; and $2,>25 \%)$. The final score of each sample is the summarization of the three parameters.

Transmission electron microscope. A portion of liver $\left(\sim 1 \mathrm{~mm}^{3}\right)$ from control and experimental groups of mice were fixed in $3 \%$ glutaraldehyde in sodium phosphate buffer $(200 \mathrm{mM}$, $\mathrm{pH}$ 7.4) for $3 \mathrm{~h}$ at $4^{\circ} \mathrm{C}$. Tissue samples were washed with the same buffer, post-fixed in $1 \%$ osmium tetroxide and sodium phosphate buffer (200 mM, pH 7.4) for $1 \mathrm{~h}$ at $4^{\circ} \mathrm{C}$. The samples were again washed with the same buffer for $3 \mathrm{~h}$ at $4^{\circ} \mathrm{C}$, dehydrated with graded series of ethanol and embedded in araldite. Thin sections were cut with LKBUM4 ultramicrotome using a diamond knife (Diatome Ltd., Nidau, Switzerland), mounted on a copper grid and stained with $2 \%$ uranyl acetate and Reynolds lead citrate. The grids were examined under a Hitachi H-7650 transmission electron microscope (Hitachi, Ltd., Tokyo, Japan).

Statistical analysis. All data are expressed as the means \pm standard deviation of at least three independent determinations for each experiment. Statistical analyses were performed using SPSS version 13.0 (SPSS, Inc., Chicago, IL, USA). A one-way analysis of variance (ANOVA) with Duncan's multiple range test was used to examine differences between groups.

\section{Results}

Hypoglycemic and lipid metabolic effects of HZL. Hypoglycemic effect of HZL on the body weight, fasting blood sugar levels and insulin levels of normal and diabetic mice was presented in Fig. 1. As shown in Fig. 1A-E, HHZL did not cause a significant $(\mathrm{P}>0.05)$ change in body weight, blood sugar levels and insulin levels in normal mice. Body weight, fasting blood glucose levels and insulin levels in the mice with HFD-alloxan-induced diabetes were significantly increased 
A

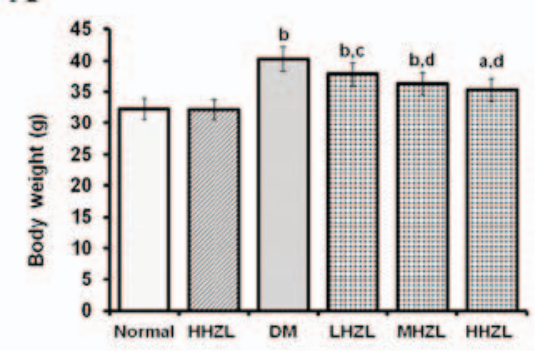

D

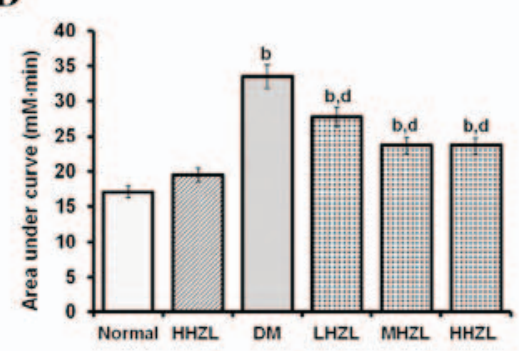

G

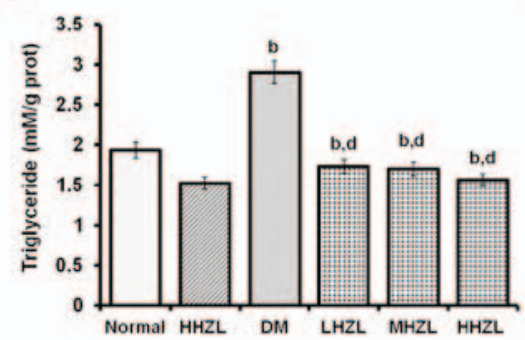

B

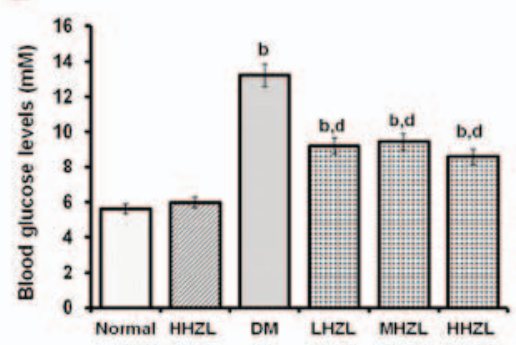

E

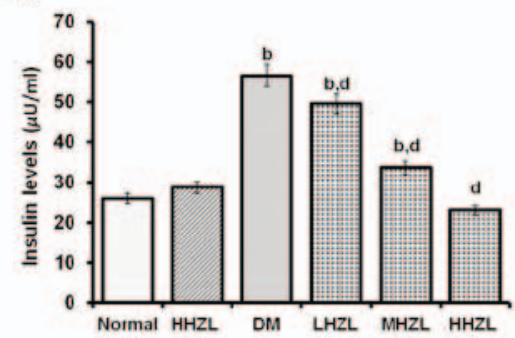

H

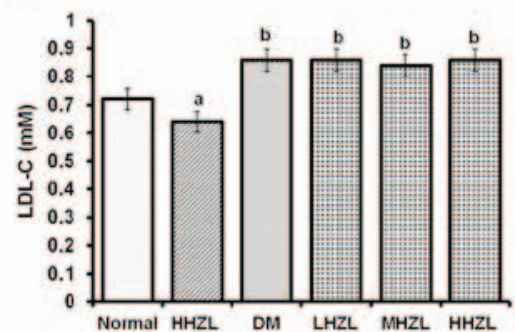

C

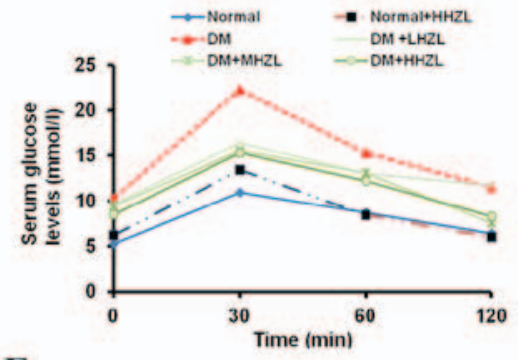

F

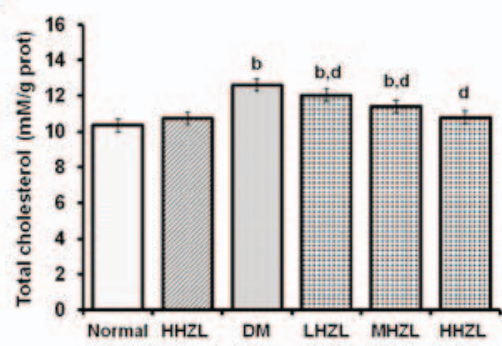

I

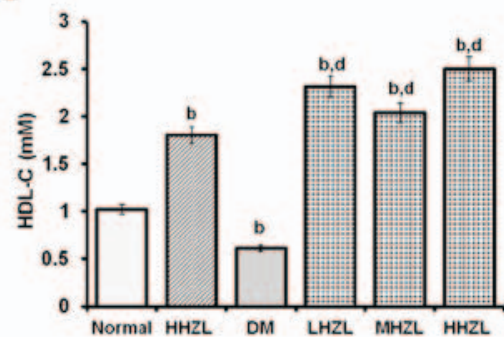

Figure 1. Hypoglycemic and lipid metabolic effects of HZL on diabetic mice. (A) Body weight; (B) blood glucose levels; (C) OGTT; (D) AUC; (E) insulin levels; (F) TC; (G) TG; (H) LDL-C levels; and (I) HDL-C levels. Data are presented as mean \pm standard deviation. ${ }^{\text {a }} \mathrm{P}<0.05$, ${ }^{\mathrm{b}} \mathrm{P}<0.01 \mathrm{vs}$. normal group; ${ }^{\mathrm{P}} \mathrm{P}<0.05$, ${ }^{\mathrm{d}} \mathrm{P}<0.01$ vs. DM group. HZL, hyperoside from Z. bungeanum leaves; OGTT, oral glucose tolerance test; AUC, area under the curve; TC, total cholesterol; TG, triglyceride; DM, diabetes mellitus; LHZL, DM + 50 mg/kg BW/day HZL; MHZL, DM + 100 mg/kg BW/day HZL; HHZL, DM + 200 mg/kg BW/day HZL; LDL-C, low-density lipoprotein cholesterol; HDL-C, high-density lipoprotein cholesterol.

$(\mathrm{P}<0.01, \mathrm{P}<0.01$ and $\mathrm{P}<0.01$, respectively) in comparison to the normal mice during 6 weeks. The administration of 200 , 100 and $50 \mathrm{mg} / \mathrm{kg}$ BW HZL (HHZL, MHZL and LHZL) in the diabetic groups led to a significant decrease $(\mathrm{P}<0.01$, $\mathrm{P}<0.01$ and $\mathrm{P}<0.05$, respectively) in body weight as compared with untreated diabetic mice in 6 weeks. In serum glucose level, administration HHZL, MHZL and LHZL in diabetic treated groups lead to significant decrease $(\mathrm{P}<0.01, \mathrm{P}<0.01$ and $\mathrm{P}<0.01$, respectively) as compared with untreated diabetic mice at 6 weeks. In OGTT, compared with diabetic mice, HZL lowered the blood glucose after glucose loading and reduced the AUC (Fig. 1D), indicating that HZL improved the glucose tolerance. Also, a noticeable decrease $(\mathrm{P}<0.01, \mathrm{P}<0.01$ and $\mathrm{P}<0.01$, respectively) was observed in serum insulin level in HHZL, MHZL and LHZL groups than the diabetic treated group at 6 weeks. Fig. 1F-I showed the mean values of TC, TG, LDL-C and HDL-C levels of both control and experimental groups after 6 weeks. An increased HDL-C level $(\mathrm{P}<0.01)$ and a decreased LDL-C level $(\mathrm{P}<0.05)$ were observed in HHZL-treated normal mice, albeit with marginal change of TC and TG levels ( $\mathrm{P}>0.05)$. HFD-alloxan-treatment resulted in obvious development of DM in mice, characterized by elevated blood TC, TG and LDL-C level $(\mathrm{P}<0.01, \mathrm{P}<0.01$ and $\mathrm{P}<0.01$, respectively), and decreased HDL-C level $(\mathrm{P}<0.01)$.
The mice with HFD-alloxan-induced diabetes that were treated with HHZL, MHZL and LHZL exhibited lowered TC, TG $(\mathrm{P}<0.01, \mathrm{P}<0.01 \mathrm{P}>0.05$ for $\mathrm{TC} ; \mathrm{P}<0.01, \mathrm{P}<0.01, \mathrm{P}<0.01$ for $\mathrm{TG}$, respectively) and largely restored HDL-C levels $(\mathrm{P}<0.01, \mathrm{P}<0.01$ and $\mathrm{P}<0.01$, respectively), compared with the untreated diabetic mice. In all HZL treatment groups, no significant change was observed in LDL-C levels $(\mathrm{P}>0.05)$.

Effects of HZL on hepatic dysfunction, oxidative stress markers and antioxidant enzymes. Fig. 2A-C present the mean values of $\mathrm{Na}^{+} / \mathrm{K}^{+}$ATPase, AST and ALT activities in the livers of mice in both normal and experimental groups 6 weeks post-treatment. HHZL treatment markedly attenuated hepatic dysfunction in diabetic animals, as evidenced by the lowering of the elevated levels of AST and ALT $(\mathrm{P}<0.01$, $\mathrm{P}<0.01$, respectively) and the restoration of the undermined $\mathrm{Na}^{+} / \mathrm{K}^{+}$ATPase activities $(\mathrm{P}<0.01)$. Fig. $2 \mathrm{D}-\mathrm{F}$ present the mean values of oxidative stress markers in both normal and experimental groups after 6 weeks in the liver. In diabetic mice the MDA levels, NO contents and iNOS activity were significantly increased $(\mathrm{P}<0.01, \mathrm{P}<0.01$ and $\mathrm{P}<0.01$, respectively). Treatment with HZL significantly reversed the MDA and NO productions and iNOS activity in diabetic mice. In the present study, HFD-alloxan induction caused an obvious loss 
A
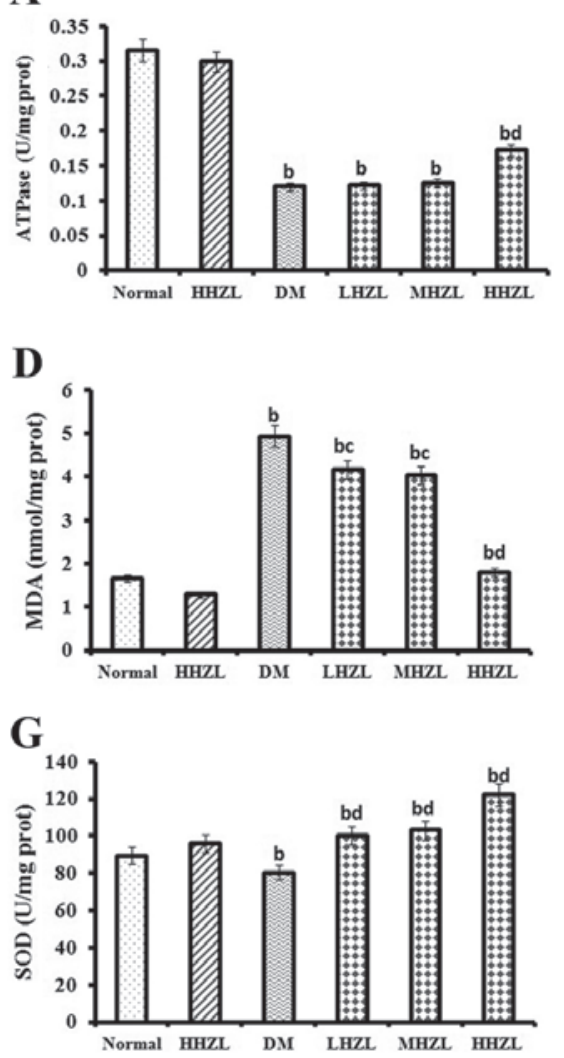

B

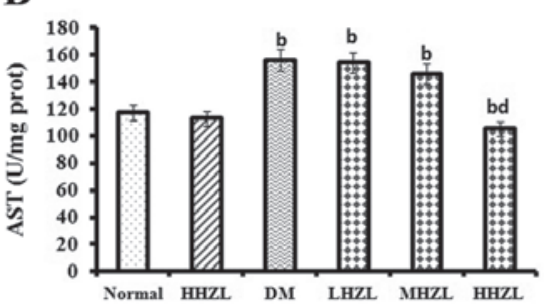

E

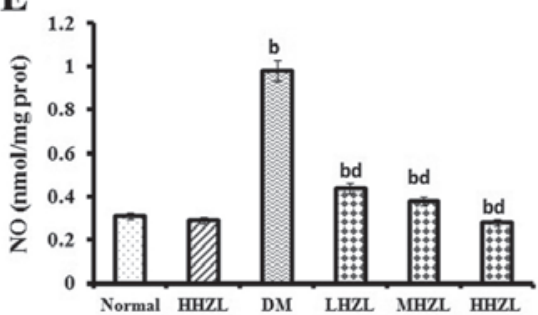

H

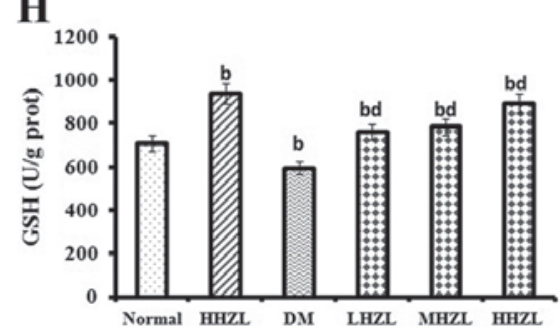

C

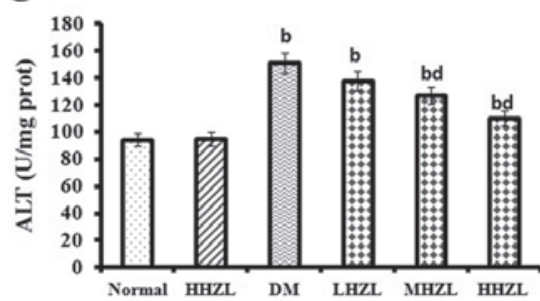

F

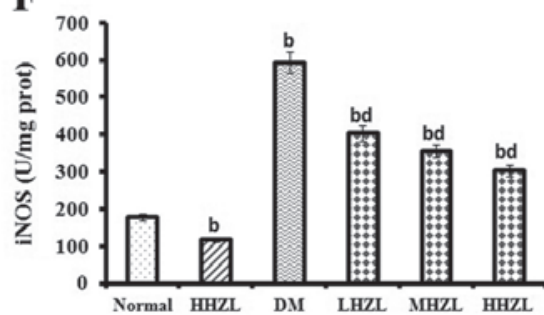

I

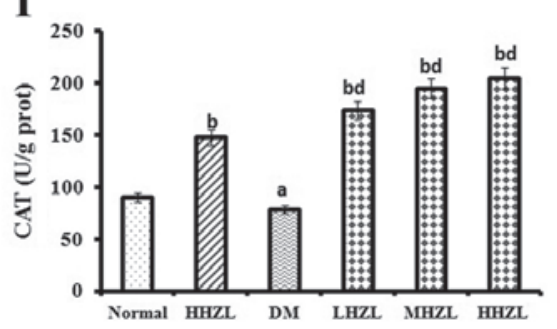

Figure 2. Effects of HZL on hepatic dysfunction, oxidative stress markers and antioxidant enzyme activity in the liver of normal and diabetic mice. (A) $\mathrm{Na}^{+} / \mathrm{K}^{+}$ATPase activity; (B) AST activity; (C) ALT activity; (D) MDA level; (E) NO level; (F) iNOS activity; (G) SOD activity; (H) GSH activity; and (I) CAT activity. Data are presented as mean \pm standard deviation. ${ }^{a} \mathrm{P}<0.05,{ }^{b} \mathrm{P}<0.01$ vs. normal group; ${ }^{\mathrm{c}} \mathrm{P}<0.05$, ${ }^{\mathrm{d}} \mathrm{P}<0.01 \mathrm{vs}$. DM group. HZL, hyperoside from Z. bungeanum leaves; AST, aspartate aminotransferase; ALT, alanine aminotransferase; MDA, malondialdehyde; NO, nitric oxide; iNOS, inducible nitric oxide synthase; SOD, superoxide dismutase; GPx, glutathione peroxidase.

in antioxidant enzyme (SOD, GPx and SOD) activities in the liver, a representative sign of DM. Nevertheless, HZL treatment markedly increased the activities of SOD, GSH and CAT $(\mathrm{P}<0.01, \mathrm{P}<0.01$ and $\mathrm{P}<0.05$, respectively) in a dose-dependent manner (Fig. 2G-I).

Hepatic apoptosis-related protein expressions. Following this, the authors determined the mechanisms behind the protective effects of HZL against HFD-alloxan-induced damage to liver. As shown in Fig. 3, HFD-alloxan treatment caused significantly increase in ATF3, p-p65, p-p38, p-Erk1/2 and p-JNK protein levels $(\mathrm{P}<0.01, \mathrm{P}<0.01, \mathrm{P}<0.01, \mathrm{P}<0.01$ and $\mathrm{P}<0.01$, respectively) compared with the normal mice. Whereas administration of HZL cause dose-dependent decrease the protein levels of ATF3 (Fig. 3A and B), p-p65, p-p38, p-Erk1/2 and p-JNK (Fig. 3C-G) compared with the untreated diabetic mice. Those results were consistent with the change of p65, p38, Erk1/2 and JNK expression levels.

Being considered to be one of meaningful events during hepatic damage caused by DM, apoptosis of hepatocytes was examined in the present study. Analysis of caspase-apoptosis pathway revealed that treatment with HFD-alloxan led to a marked increase in Bax and Cyt c, and a decrease in Bcl-2 levels, and thus enhanced the cleavage of caspase- 9 and caspase-3 $(\mathrm{P}<0.01, \mathrm{P}<0.01, \mathrm{P}<0.01, \mathrm{P}<0.01$ and $\mathrm{P}<0.01$, respectively; Fig. 4). Not surprisingly, HZL treatment markedly decreased apoptotic signaling by lowering the protein levels of Bax (Fig. 4C), Cyt c (Fig. 4E and F), elevating expression of Bcl-2 (Fig. 4A and B) and inhibiting cleavage of caspase-9 (Fig. 4D) and caspase-3 (Fig. 4G and H).

Histopathological analysis. The liver tissue sections indicated that healthy control mice had normal hepatic cells with well-preserved cytoplasm, nucleus and central vein (Fig. 5). In the diabetic group, the loss of hepatic architecture was observed, accompanied by lymphocytic inflammation and focal necrosis of hepatic cells. HZL treatment markedly restored the structural integrity of the damaged cells. Nevertheless, total loss of hepatic architecture and partially lymphocytic inflammation was observed in LHZL and MHZL group. Overall, status of liver tissues in the HHZL group was better than the LHZL and MHZL group and had almost returned to the normal level (Fig. 5A and B).

The ultrastructural changes occurred in hepatic of mice are presented in Fig. 6. Fig. 6A represents the EM image of hepatic of control mice, showing the intact cellular organelles such as mitochondria, endoplasmic reticulum (ER) and Golgi complex, and HZL treatment alone no obvious influence on hepatocytes (Fig. 6B). The EM image of hepatocytes from diabetic mice (Fig. 6C) exhibited a noticeable destruction of hepatic accompanied with loss of mitochondrial cristae, vacuolization with ballooning appearance of mitochondria, as well as dilation of the rough ER, which could be considerably prevented by HZL treatment during the progression of DM 
A

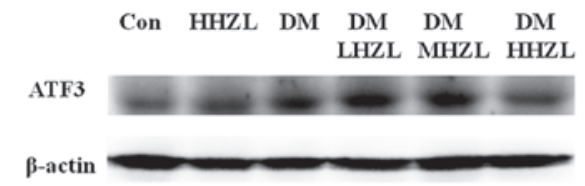

B

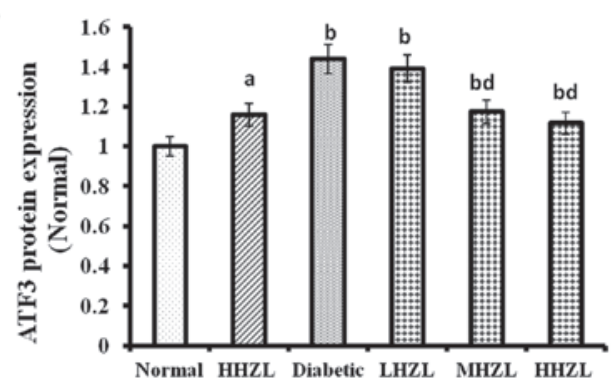

D

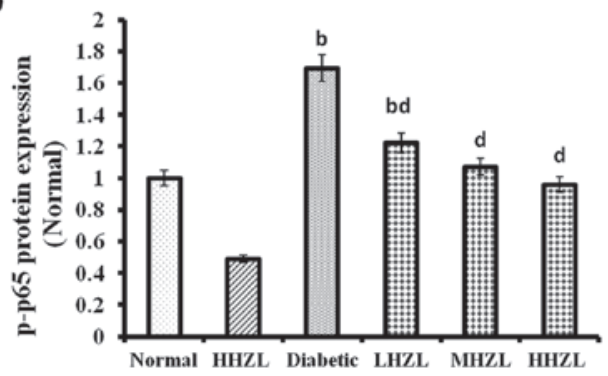

F

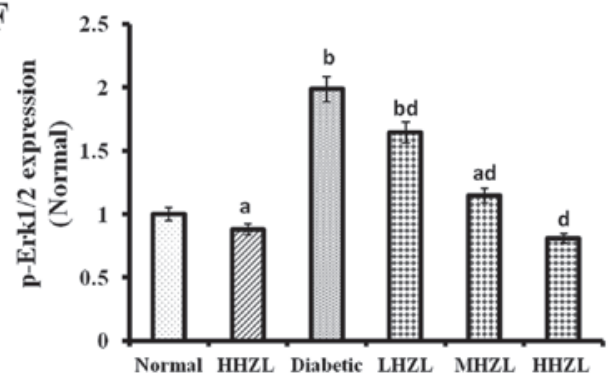

C

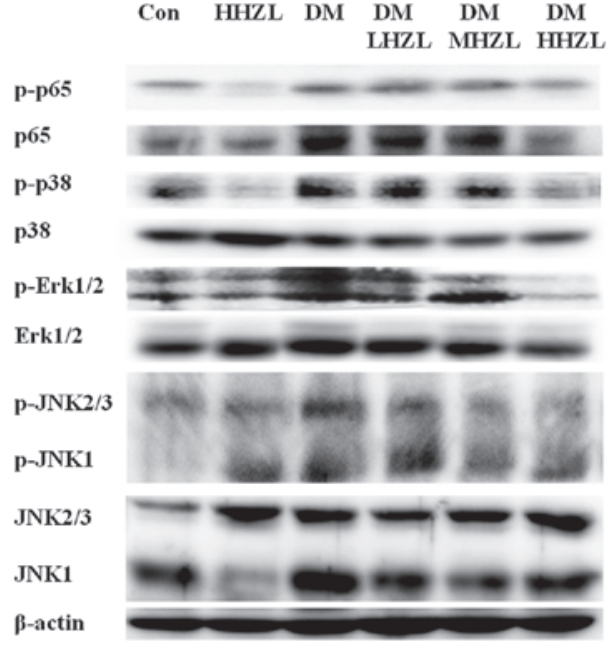

$\mathbf{E}$

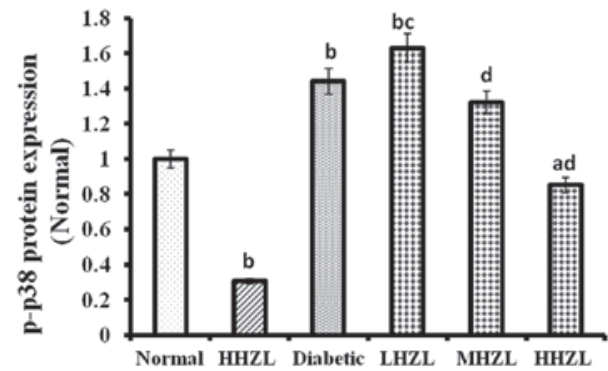

G

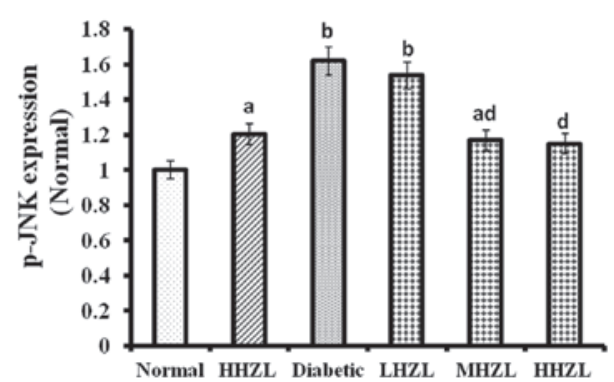

Figure 3. The protein expression of ATF3 and the phosphorylation p65, p38, ERK1/2 and JNK1/2/3 in the liver of normal and diabetic mice. (A and C) Western blotting and quantitative analysis of renal (B) ATF3; (D) p-p65; (E) p-p38; (F) p-Erk1/2; and (G) p-JNK expression. Data are presented as mean \pm standard deviation. ${ }^{\text {a }} \mathrm{P}<0.05$, ${ }^{\mathrm{b}} \mathrm{P}<0.01$ vs. normal group; ${ }^{\mathrm{c}} \mathrm{P}<0.05$, ${ }^{\mathrm{d}} \mathrm{P}<0.01$ vs. DM group. ATF3, activating transcription factor 3 ; HZL, hyperoside from $Z$. bungeanum leaves; DM, diabetes mellitus; LHZL, DM + 50 mg/kg BW/day HZL; MHZL, DM + 100 mg/kg BW/day HZL; HHZL, DM + 200 mg/kg BW/day HZL; Con, control.

induced by HFD-alloxan, as evidenced by minimal nuclear membrane damage, minimal loss in the cristae with weak swelling of mitochondria and no vacuolarization of cytoplasmic region of hepatic (Fig. 6D).

\section{Discussion}

DM is currently considered a worldwide epidemic and finding effective therapeutic strategies against this disease is highly important. In the present study, the authors investigated the effects of the potent antioxidants hyperoside isolated from $Z$. bungeanum leaves in mice with HFD-alloxan-induced diabetes. The daily administration of HHZL (200 mg/kg BW) for 42 days led to significant decline in LDL-C levels, iNOS activity, cleavage of caspase-9 and caspase-3, as well as evident increases in HDL-C levels, GPx activity, CAT activity and protein levels of ATF3 comparison with normal groups. Insignificant difference in body weight gain, blood glucose levels, insulin levels, TC levels, $\mathrm{Na}^{+} / \mathrm{K}^{+}$ ATPase activity, AST activity, ALT activity, MDA levels, NO levels, SOD activity, as well as phosphorylation of p65, p38 and Erk1/2 were observed. These results indicated that hyperoside isolated from $Z$. bungeanum leaves was no toxic effects on normal mice.

A previous study demonstrated that hyperoside can decrease blood glucose levels in streptozotocin-induced hyperglycemia by improving the function of pancreatic islets and increasing glycolysis and decreasing gluconeogenesis (22). In the present study, HFD-alloxan led to a significant increase in body weight, blood glucose levels and insulin levels in the experimental mice. Body weight gain, blood glucose levels and insulin levels were reduced in the HZL-treated groups in a dose-dependent manner when compared with the diabetic mice. These results supported the notion that HZL may be developed as an anti-hyperglycemic agent for the treatment of DM. 
A

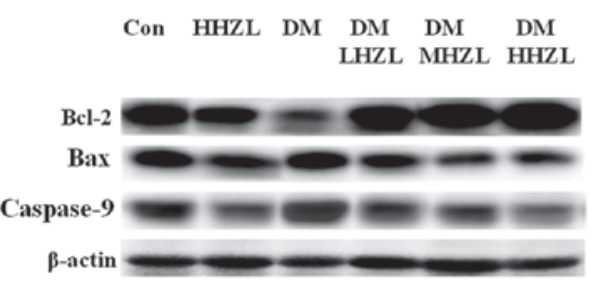

C

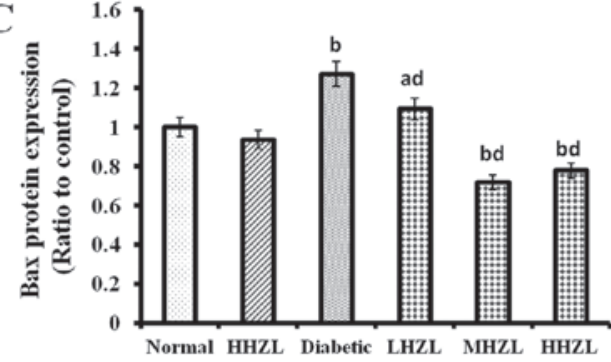

E

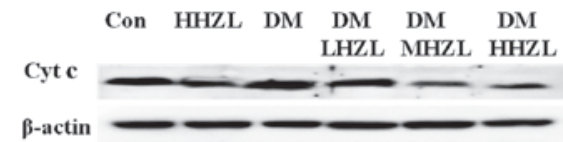

F

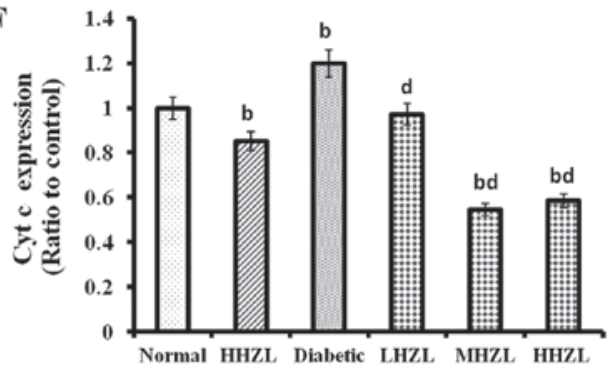

B

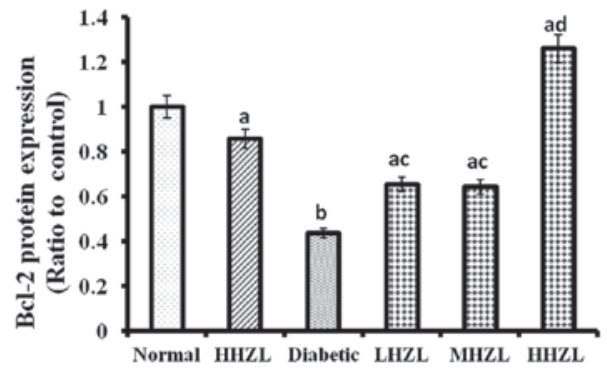

D

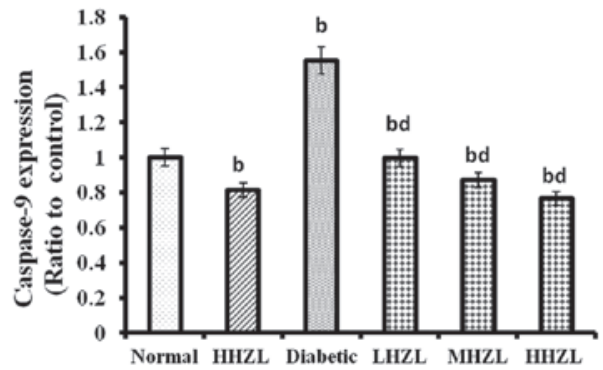

G

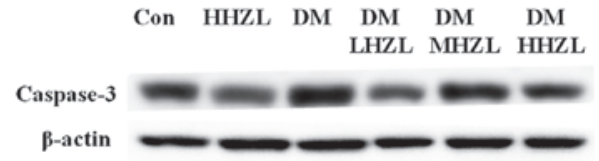

H

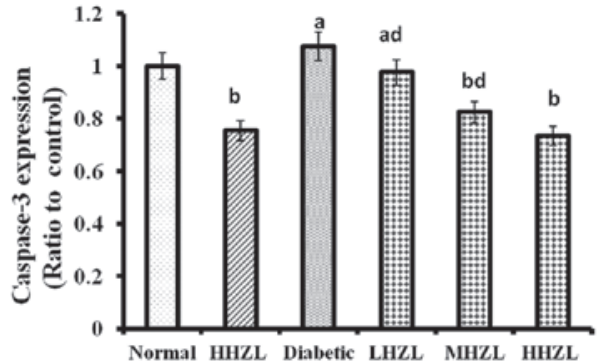

Figure 4. Bcl-2, Bax, Cyt c, caspase-9 and caspase-3 protein expression in the liver of normal and diabetic mice. (A, E and G) Western blotting and quantitative analysis of renal (B) Bcl-2; (C) Bax; (D) caspase-9; (F) Cyt c; and (H) caspase-3 expression. Data are presented as mean \pm standard deviation. ${ }^{\text {a }} \mathrm{P}<0.05$, ${ }^{b} \mathrm{P}<0.01$ vs. normal group; ${ }^{\mathrm{P}}<0.05,{ }^{\mathrm{d}} \mathrm{P}<0.01$ vs. DM group. HZL, hyperoside from Z. bungeanum leaves; DM, diabetes mellitus; LHZL, DM + 50 mg/kg BW/day HZL; MHZL, DM + 100 mg/kg BW/day HZL; HHZL, DM + 200 mg/kg BW/day HZL; Con, control.

The mice with HFD-alloxan-induced diabetes showed abnormalities in lipid metabolism, as evidenced by increased TG, TC and LDL-C levels and decreased HDL-C levels, similar to the characteristics of human type 2 diabetes $(24,25)$. Hypertriglyceridemia may occur due to increased absorption and formation of triglycerides in the form of chylomicrons following consumption of a diet rich in fat or through increased endogenous production of TG-enriched hepatic VLDL and decreased TG uptake in peripheral tissues (26). In the present study, a decrease in TC, TG and LDL-C levels and an increase in HDL-C levels were observed in the serum of HZL-treated diabetic mice in a dose-dependent manner, suggesting that HZL regulated hepatic lipid metabolism.

In experimental diabetes, alloxan exerts its toxic effects on liver and other organs in addition to pancreatic $\beta$-cells $(27,28)$. The higher levels of ALT observed in the diabetic mice indicates a certain degree of hepatic damage. In this study, the diabetic hyperglycemia produced elevation of hepatic AST and ALT levels, which were considered as typical signs of liver dysfunction. HHZL decreased the elevated levels of AST and ALT in diabetic mice. In experimental diabetes, changes in $\mathrm{Na}^{+} / \mathrm{K}^{+}$ATPase activity have been reported in different tissues. The diabetic mice had significantly decreased activities of $\mathrm{Na}^{+} / \mathrm{K}^{+}$ATPase in erythrocytes and tissues $(29,30)$. A significant increase in $\mathrm{Na}^{+} / \mathrm{K}^{+}$ATPase activity was observed in HZL-treated diabetic mice. These results, concomitant with the effect on histological changes, indicated that HZL protects against diabetic liver injury.

Oxidative stress is considered to be the main factor contributing to development of diabetic complications and tissue injury (31). The insulin insufficiency and hyperglycemia further augment liver damage through ROS mediated lipid peroxidation of hepatocellular membrane $(32,33)$. Hepatic $\mathrm{Na}^{+} / \mathrm{K}^{+}$ATPase activity was reduced, which may also be due to the membrane peroxidative damage induced by increased lipid peroxidation status (34). In diabetic mice, the authors observed a significant increase in MDA levels, whereas HZL treatment significantly reduced MDA levels in diabetic mice. Excess NO by iNOS has been reported to induce deleterious effects in the liver (35). These data showed that treatment with HZL caused 


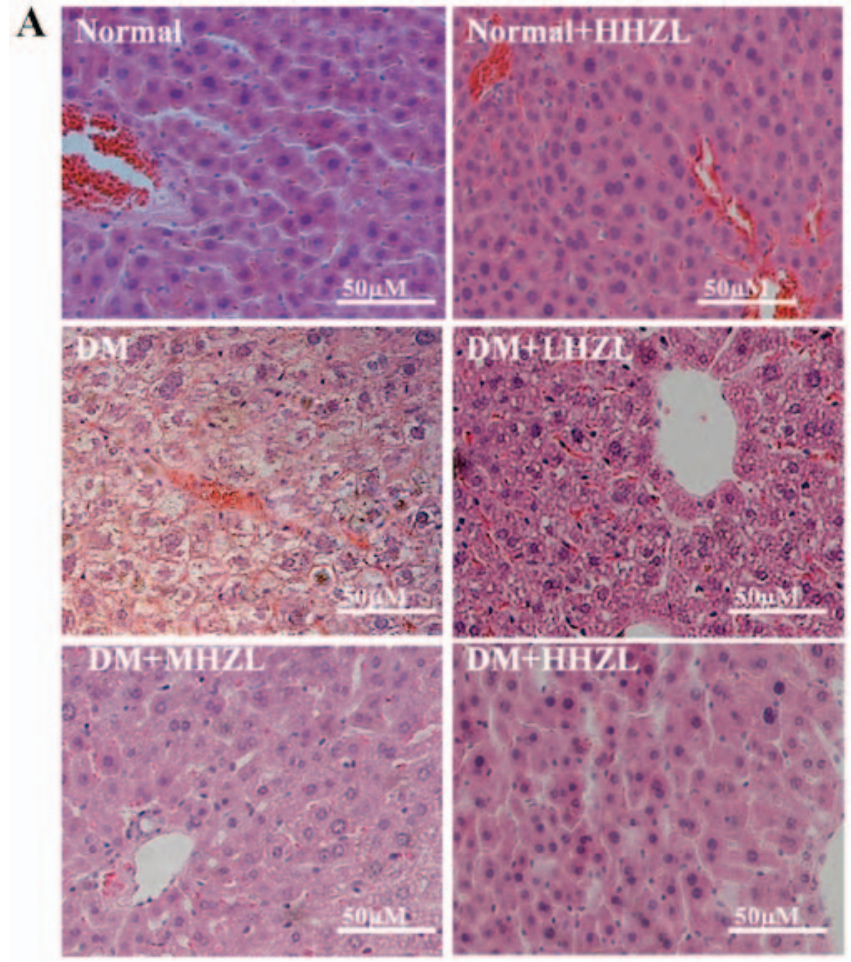

B

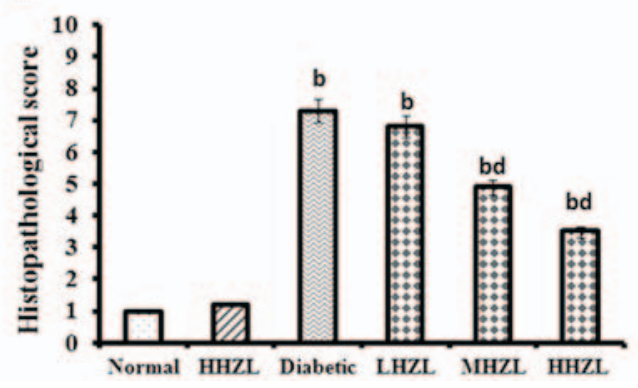

Figure 5. HZL attenuates diabetic liver injury in mice. (A) A representative figure of hepatic morphological changes using hematoxylin and eosin staining (magnification, x200); (B) the graph shows the quantitative analysis of inflammatory cell infiltration. Data are presented as mean \pm standard deviation. ${ }^{\mathrm{b}} \mathrm{P}<0.01$ vs. normal group; ${ }^{\mathrm{d}} \mathrm{P}<0.01$ vs. DM group. HZL, hyperoside from Z. bungeanum leaves; DM, diabetes mellitus; LHZL, DM +50 mg/kg BW/day HZL; MHZL, DM + 100 mg/kg BW/day HZL; HHZL, DM + 200 mg/kg BW/day HZL.

marked reduction in NO production and iNOS activity in diabetic liver. These results indicated that the protective effect of HZL be related to antioxidant activity.

Antioxidant therapy is considered to be a significant pharmacological prelude for the management of diabetes, as their benefits are not only attributed to its radical quenching but also to their ability to interact with numerous basic cellular activities. In this context, several studies have reported the declined activities of these antioxidant enzymes in the diabetic liver and HZL with antioxidant activities has been shown to restore activities of these antioxidant enzymes in liver of diabetic mice. These findings suggested that the HZL may exert its hepatic protect effect through the enhancement of cellular antioxidant system.

ATF3 is an adaptive response transcription factor for various cell types to cope with extra and/or intracellular changes, including cytokines, chemokines, growth factors, hormones,
A Normal

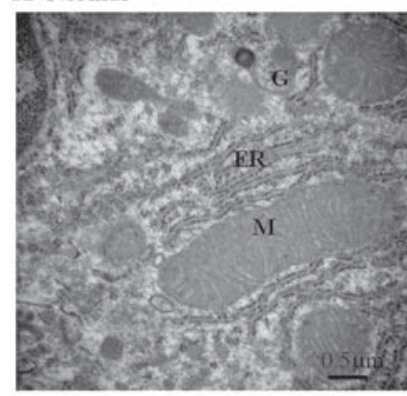

C DM

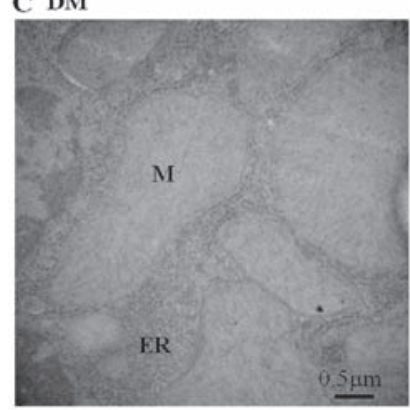

B Normal + HHZI

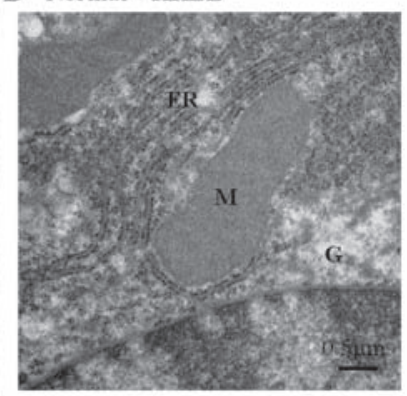

D $\mathrm{DM}+\mathrm{HHZL}$

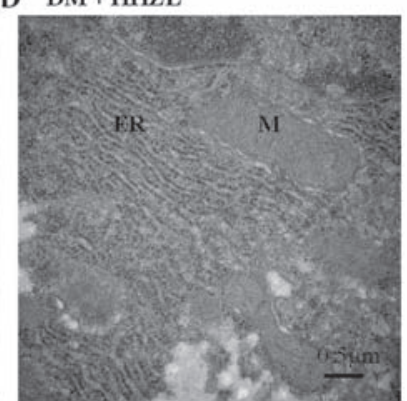

Figure 6. Transmission electron micrographs of hepatocytes of normal and diabetic mice (magnification, x15,000). (A) Normal; (B) normal + HHZL; (C) diabetic; and (D) diabetic + HHZL. HHZL, DM + $200 \mathrm{mg} / \mathrm{kg}$ BW/day hyperoside from $Z$. bungeanum leaves. ER, endoplasmic reticulum; M, mitochondria.

hypoxia, DNA damage and nutrient deprivation. Expression of its corresponding gene is induced by oxidative stress signals in a variety of tissues, including the liver (36). ATF3 has been proven to regulate apoptotic cell death in response to oxidant stress through suppressing NF- $\mathrm{B}$-dependent transcription of anti-apoptotic genes $(37,38)$, triggering the MAPKs $(39,40)$, mediating the caspase activation (41). Researchers also have demonstrated that oxidative stress mediates the activation of MAPK, NF- $\kappa \mathrm{B}$ and caspase-dependent signaling pathways (42). Overexpression of activated NF- $\kappa$ B, MAPK and caspase proteins is considered as an important factor contributing to the development of diabetic liver injury (43-45). $\mathrm{NF}-\kappa \mathrm{B}$, as an oxidative stress-responsive transcription factor, enhances the inducible nitric oxide synthase expression leading to the elevated generation of NO (46). Meanwhile, $\mathrm{NF}-\kappa \mathrm{B}$ is an appropriate target to treat hepatocellular toxicity (47). ROS exert a significant effect on hepatocyte apoptosis in diabetes (48). Literature also suggests that extensive cell apoptosis eventually resulting in loss of function in tissues is associated with upregulation of Bax, and release of Cyt c (49). Cyt c released from mitochondria leads to activation of caspase- 9 that induced apoptosis, which has been implicated in the pathogenesis of diabetic liver injury. For all these reasons, the authors evaluated the phosphorylation of p65/NF- $\kappa$ B, MAPK (including p38, JNK and ERK), expression of apoptosis related proteins such as Bcl-2, Bax, Cyt c and activation of caspase- 9 and caspase- 3 in diabetic induced liver injury and evaluated the inhibitory effects of HZL on these risk factors. The results of western blot analysis revealed that HFD-alloxan treatment promoted the phosphorylation of p65, p38, JNK and ERK proteins, whereas HZL administration decreased this phosphorylation. These results indicated that 
the protect effects of HZL may be associated with the suppression of NF- $\mathrm{KB}$ and MAPK signaling pathways in the livers of diabetic mice. Furthermore, HZL treatment of diabetic mice significantly suppressed hepatic protein levels of Bax, Cyt c, caspase- 9 and caspase-3, although there were no changes in Bcl-2 protein levels among all experimental groups. The results presented here suggested that HZL could prevent apoptosis-induced hepatic damage, at least in part, through the amelioration of oxidative stress-induced diabetic liver injury.

Recently, the effect of hyperoside on alleviating diabetes and diabetic complications has been receiving increasing attention (50-52). The authors further studied the effect of HZL in islet cells, and results show that HZL could inhibit HFD-alloxan-induced islet cells injury by downregulating p65/NF- $\kappa \mathrm{B}$ and ERK/MAPK signals and inhibiting apoptosis (unpublished data). In addition, a study by Martin et al (53) showed that Cocoa phenolic extract including hyperoside protects pancreatic $\beta$-cells against oxidative stress. Instreptozotocin-induced type 2 diabetic rats, hyperoside could directly lower glucose levels by improving the function of pancreatic islets and increasing glycolysis and decreasing gluconeogenesis (22). Moreover, many studies have shown that quercetin as aglycone of hyperoside prevented and protected streptozotocin-induced oxidative stress and $\beta$-cell damage in the rat pancreas (54-57). These results theoretically support the notion that HZL protect the islet cells from the injury caused by oxidative stress. Furthermore, studies on the effect of HZL in islet cells are required to get a deeper understanding of molecular mechanisms of the HZL hyperglycemic effect.

In conclusion, the present study demonstrated that hyperosides from $Z$. bungeanum leaves exerted significant anti-hyperglycemic and hepatocyte-protective effects in mice with HFD- and alloxan-induced diabetes. The observed hepatocyte protection, as well as the antioxidant potential of hyperoside was partially responsible for its anti-diabetogenic properties. Its hepatocyte-protective mechanisms involved the inhibition of the NF- $\kappa$ B, MAPK and caspase-dependent apoptotic pathways. These maybe helpful to understand the role of $Z$. bungeanum leaves in the clinical treatment of DM and its secondary complications.

\section{Acknowledgements}

The present study was financed by the Special Fund for Forest Scientific Research in the Public Welfare (grant no. 201304811), the National Natural Science Foundation of China (grant no. 31101266) and the Scientific and Technical Foundation of Shaanxi Province (grant no. 2014JM41005). The present study was partly supported by the Institute of Mitochondrial Biology and Medical, Xi'an Jiaotong University (Xi'an, China).

\section{References}

1. Bullon P, Newman HN and Battino M: Obesity, diabetes mellitus, atherosclerosis and chronic periodontitis: a shared pathology via oxidative stress and mitochondrial dysfunction? Periodontol 2000 64: 139-153, 2014.

2. Crujeiras AB, Díaz-Lagares A, Carreira MC, Amil M and Casanueva FF: Oxidative stress associated to dysfunctional adipose tissue: a potential link between obesity, type 2 diabetes mellitus and breast cancer. Free Radic Res 47: 243-256, 2013.
3. Stefanović A, Kotur-Stevuljević J, Spasić S, Bogavac-Stanojević N and Bujisić N: The influence of obesity on the oxidative stress status and the concentration of leptin in type 2 diabetes mellitus patients. Diabetes Res Clin Pract 79: 156-163, 2008.

4. Shima T, Uto H,Ueki K, Takamura T, Kohgo Y, Kawata S, Yasui K, Park H, Nakamura N, Nakatou T, et al: Clinicopathological features of liver injury in patients with type 2 diabetes mellitus and comparative study of histologically proven nonalcoholic fatty liver diseases with or without type 2 diabetes mellitus. J Gastroenterol 48: 515-525, 2013.

5. Takeuchi J, Takada A, Nakada Y, Sawae G and Okumura Y: Clinical and experimental studies of liver injury in diabetes mellitus. II. Experimental studies. Acta Hepatosplenol 17: 228-240, 1970.

6. Casas-Grajales S and Muriel P: Antioxidants in liver health. World J Gastrointest Pharmacol Ther 6: 59-72, 2015.

7. Shin SM, Yang JH and Ki SH: Role of the Nrf2-ARE pathway in liver diseases. Oxid Med Cell Longev 2013: 763257, 2013.

8. Stadler K, Jenei V, von Bölcsházy G, Somogyi A and Jakus J: Increased nitric oxide levels as an early sign of premature aging in diabetes. Free Radic Biol Med 35: 1240-1251, 2003.

9. Lucchesi AN, Freitas NT, Cassettari LL, Marques SF and Spadella CT: Diabetes mellitus triggers oxidative stress in the liver of alloxan-treated rats: a mechanism for diabetic chronic liver disease. Acta Cir Bras 28: 502-508, 2013.

10. Liu X, Zhang J, Ming Y, Chen X, Zeng M and Mao Y: The aggravation of mitochondrial dysfunction in nonalcoholic fatty liver disease accompanied with type 2 diabetes mellitus. Scand J Gastroenterol 50: 1152-1159, 2015.

11. Xiong QB and Shi DW: Morphological and histological studies of Chinese traditional drug 'hua jiao' (pericarpium zanthoxyli) and its allied drugs. Yao Xue Xue Bao 26: 938-947, 1991 (In Chinese).

12. Yang LC, Li R, Tan J and Jiang ZT: Polyphenolics composition of the leaves of Zanthoxylum bungeanum Maxim. grown in Hebei, China, and their radical scavenging activities. J Agric Food Chem 61: 1772-1778, 2013.

13. Zhang Y, Luo Z, Wang D, He F and Li D: Phytochemical profiles and antioxidant and antimicrobial activities of the leaves of Zanthoxylum bungeanum. ScientificWorldJournal 2014: 181072, 2014.

14. Zhang Y, Luo Z and Wang D: Efficient quantification of the phenolic profiles of Zanthoxylum bungeanum leaves and correlation between chromatographic fingerprint and antioxidant activity. Nat Prod Res 29: 2024-2029, 2015.

15. Zhang Y, Wang D, Yang L, Zhou D and Zhang J: Purification and characterization of flavonoids from the leaves of Zanthoxylum bungeanum and correlation between their structure and antioxidant activity. PLoS One 9: e105725, 2014.

16. Sukito A and Tachibana S: Isolation of hyperoside and isoquercitrin from Camellia sasanqua as antioxidant agents. Pak J Biol Sci 17: 999-1006, 2014.

17. Li FR, Yu FX, Yao ST, Si YH, Zhang W and Gao LL: Hyperin extracted from Manchurian rhododendron leaf induces apoptosis in human endometrial cancer cells through a mitochondrial pathway. Asian Pac J Cancer Prev 13: 3653-3656, 2012.

18. Ku SK, Kwak S, Kwon OJ and Bae JS: Hyperoside inhibits highglucose-induced vascular inflammation in vitro and in vivo. Inflammation 37: 1389-1400, 2014.

19. Ku SK, Kim TH, Lee S, Kim SM and Bae JS: Antithrombotic and profibrinolytic activities of isorhamnetin-3-O-galactoside and hyperoside. Food Chem Toxicol 53: 197-204, 2013.

20. Li ZL, Hu J, Li YL, Xue F, Zhang L, Xie JQ, Liu ZH, Li H, Yi DH, Liu JC, et al: The effect of hyperoside on the functional recovery of the ischemic/reperfused isolated rat heart: potential involvement of the extracellular signal-regulated kinase 1/2 signaling pathway. Free Radic Biol Med 57: 132-140, 2013.

21. Jung HA, Islam MD, Kwon YS, Jin SE, Son YK, Park JJ, Sohn HS and Choi JS: Extraction and identification of three major aldose reductase inhibitors from Artemisia montana. Food Chem Toxicol 49: 376-384, 2011.

22. Verma N, Amresh G, Sahu PK, Mishra N, Rao ChV and Singh AP: Pharmacological evaluation of hyperin for antihyperglycemic activity and effect on lipid profile in diabetic rats. Indian J Exp Biol 51: 65-72, 2013.

23. Liu X, Zhu L, Tan J, Zhou X, Xiao L, Yang X and Wang B: Glucosidase inhibitory activity and antioxidant activity of flavonoid compound and triterpenoid compound from Agrimonia pilosa Ledeb. BMC Complement Altern Med 14: 12, 2014. 
24. Friedman MI: Insulin-induced hyperphagia in alloxan-diabetic rats fed a high-fat diet. Physiol Behav 19: 597-599, 1977.

25. Tang LQ, Wei W, Chen LM and Liu S: Effects of berberine on diabetes induced by alloxan and a high-fat/high-cholesterol diet in rats. J Ethnopharmacol 108: 109-115, 2006.

26. Dixon JL, Shen S, Vuchetich JP, Wysocka E, Sun GY and Sturek M: Increased atherosclerosis in diabetic dyslipidemic swine: protection by atorvastatin involves decreased VLDL triglycerides but minimal effects on the lipoprotein profile. J Lipid Res 43: 1618-1629, 2002.

27. Buko V, Lukivskaya O, Nikitin V, Tarasov Y, Zavodnik L, Borodinsky A, Gorenshtein B, Janz B, Gundermann KJ and Schumacher R: Hepatic and pancreatic effects of polyenoylphosphatidylcholine in rats with alloxan-induced diabetes. Cell Biochem Funct 14: 131-137, 1996.

28. Zhang X, Liang W, Mao Y, Li H, Yang Y and Tan H: Hepatic glucokinase activity is the primary defect in alloxan-induced diabetes of mice. Biomed Pharmacother 63: 180-186, 2009.

29. Al-Numair KS, Veeramani C, Alsaif MA and Chandramohan G: Influence of kaempferol, a flavonoid compound, on membrane-bound ATPases in streptozotocin-induced diabetic rats Pharm Biol 53: 1372-1378, 2015.

30. Ramesh B and Pugalendi KV: Influence of umbelliferone on membrane-bound ATPases in streptozotocin-induced diabetic rats. Pharmacol Rep 59: 339-348, 2007.

31. Çelık VK, Sahın ZD, Sari İ and Bakir S: Comparison of oxidant/antioxidant, detoxification systems in various tissue homogenates and mitochondria of rats with diabetes induced by streptozocin. Exp Diabetes Res 2012: 386831, 2012.

32. Alevizos I, Misra J, Bullen J, Basso G, Kelleher J, Mantzoros C and Stephanopoulos G: Linking hepatic transcriptional changes to high-fat diet induced physiology for diabetes-prone and obese-resistant mice. Cell Cycle 6: 1631-1638, 2007.

33. Feng W, Zhao T, Mao G, Wang W, Feng Y, Li F, Zheng D, Wu H, Jin D, Yang L, et al: Type 2 diabetic rats on diet supplemented with chromium malate show improved glycometabolism, glycometabolism-related enzyme levels and lipid metabolism. PLoS One 10: e0125952, 2015.

34. Banks MA, Porter DW, Martin WG and Castranova V: Effects of in vitro ozone exposure on peroxidative damage, membrane leakage, and taurine content of rat alveolar macrophages. Toxicol Appl Pharmacol 105: 55-65, 1990.

35. Bahmani F, Tajadadi-Ebrahimi M, Kolahdooz F, Mazouchi M, Hadaegh H, Jamal AS, Mazroii N, Asemi S and Asemi Z: The consumption of synbiotic bread containing Lactobacillus sporogenes and inulin affects nitric oxide and malondialdehyde in patients with type 2 diabetes mellitus: randomized, double-blind, placebo-controlled trial. J Am Coll Nutr 35: 506-513, 2016

36. Allen-Jennings AE, Hartman MG, Kociba GJ and Hai T: The roles of ATF3 in glucose homeostasis. A transgenic mouse mode with liver dysfunction and defects in endocrine pancreas. J Biol Chem 276: 29507-29514, 2001

37. Hua B, Tamamori-Adachi M, Luo Y, Tamura K, Morioka M, Fukuda M, Tanaka Y and Kitajima S: A splice variant of stress response gene ATF3 counteracts NF-kappaB-dependent anti-apoptosis through inhibiting recruitment of CREB-binding protein/p300 coactivator. J Biol Chem 281: 1620-1629, 2006.

38. Jung DH, Kim KH, Byeon HE, Park HJ, Park B, Rhee DK, Um SH and Pyo S: Involvement of ATF3 in the negative regulation of iNOS expression and NO production in activated macrophages. Immunol Res 62: 35-45, 2015.

39. $\mathrm{Lu} \mathrm{D}$, Chen $\mathrm{J}$ and Hai T: The regulation of ATF3 gene expression by mitogen-activated protein kinases. Biochem J 401: 559-567, 2007.

40. Inoue K, Zama T, Kamimoto T, Aoki R, Ikeda Y, Kimura H and Hagiwara M: TNFalpha-induced ATF3 expression is bidirectionally regulated by the JNK and ERK pathways in vascular endothelial cells. Genes Cells 9: 59-70, 2004.

41. Mashima T, Udagawa S and Tsuruo T: Involvement of transcriptional repressor ATF3 in acceleration of caspase protease activation during DNA damaging agent-induced apoptosis. J Cell Physiol 188: 352-358, 2001
42. Ma JQ, Ding J, Zhang L and Liu CM: Ursolic acid protects mouse liver against $\mathrm{CCl} 4$-induced oxidative stress and inflammation by the MAPK/NF-кB pathway. Environ Toxicol Pharmacol 37: 975-983, 2014

43. Kohl T, Gehrke N, Schad A, Nagel M, Wörns MA, Sprinzl MF, Zimmermann T, He YW, Galle PR, Schuchmann M, et al: Diabetic liver injury from streptozotocin is regulated through the caspase- 8 homolog cFLIP involving activation of JNK2 and intrahepatic immunocompetent cells. Cell Death Dis 4: e712, 2013.

44. Sawant SP, Dnyanmote AV and Mehendale HM: Mechanisms of inhibited liver tissue repair in toxicant challenged type 2 diabetic rats. Toxicology 232: 200-215, 2007.

45. Devi SS and Mehendale HM: The role of NF-kappaB signaling in impaired liver tissue repair in thioacetamide-treated type 1 diabetic rats. Eur J Pharmacol 523: 127-136, 2005.

46. Farombi EO, Shrotriya S and Surh YJ: Kolaviron inhibits dimethyl nitrosamine-induced liver injury by suppressing COX-2 and iNOS expression via NF-kappaB and AP-1. Life Sci 84: $149-155,2009$.

47. Muriel P: NF-kappaB in liver diseases: a target for drug therapy. J Appl Toxicol 29: 91-100, 2009.

48. Bhattacharya S, Gachhui R and Sil PC: The prophylactic role of D-saccharic acid-1,4-lactone against hyperglycemia-induced hepatic apoptosis via inhibition of both extrinsic and intrinsic pathways in diabetic rats. Food Funct 4: 283-296, 2013.

49. Rashid K, Das J and Sil PC: Taurine ameliorate alloxan induced oxidative stress and intrinsic apoptotic pathway in the hepatic tissue of diabetic rats. Food Chem Toxicol 51: 317-329, 2013.

50. Zhou L, An XF, Teng SC, Liu JS, Shang WB, Zhang AH, Yuan YG and Yu JY: Pretreatment with the total flavone glycosides of flos Abelmoschus manihot and hyperoside prevents glomerular podocyte apoptosis in streptozotocin-induced diabetic nephropathy. J Med Food 15: 461-468, 2012.

51. An X, Zhang L, Yuan Y, Wang B, Yao Q, Li L, Zhang J, He M and Zhang J: Hyperoside pre-treatment prevents glomerular basement membrane damage in diabetic nephropathy by inhibiting podocyte heparanase expression. Sci Rep 7: 6413, 2017.

52. Zhang Z, Sethiel MS, Shen W, Liao S and Zou Y: Hyperoside downregulates the receptor for advanced glycation end products (RAGE) and promotes proliferation in ECV304 cells via the c-Jun N-terminal kinases (JNK) pathway following stimulation by advanced glycation end-products in vitro. Int J Mol Sci 14: 22697-22707, 2013.

53. Martín MA, Ramos S, Cordero-Herrero I, Bravo L and Goya L: Cocoa phenolic extract protects pancreatic beta cells against oxidative stress. Nutrients 5: 2955-2968, 2013.

54. Coskun O, Kanter M, Korkmaz A and Oter S: Quercetin, a flavonoid antioxidant, prevents and protects streptozotocin-induced oxidative stress and beta-cell damage in rat pancreas. Pharmacol Res 51: 117-123, 2005.

55. Adewole SO, Caxton-Martins EA and Ojewole JA: Protective effect of quercetin on the morphology of pancreatic beta-cells of streptozotocin-treated diabetic rats. Afr J Tradit Complement Altern Med 4: 64-74, 2006.

56. Youl E, Bardy G, Magous R, Cros G, Sejalon F, Virsolvy A, Richard S, Quignard JF, Gross R, Petit P, et al: Quercetin potentiates insulin secretion and protects INS-1 pancreatic $\beta$-cells against oxidative damage via the ERK1/2 pathway. Br J Pharmacol 161: 799-814, 2010.

57. Bardy G, Virsolvy A, Quignard JF, Ravier MA, Bertrand G, Dalle S, Cros G, Magous R, Richard S and Oiry C: Quercetin induces insulin secretion by direct activation of L-type calcium channels in pancreatic beta cells. Br J Pharmacol 169: 1102-1113, 2013.

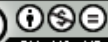

This work is licensed under a Creative Commons Attribution-NonCommercial-NoDerivatives 4.0 International (CC BY-NC-ND 4.0) License. 